NORDITA-2003/1 HE

\title{
$\mathcal{N}=1$ super Yang-Mills theories and wrapped branes
}

\author{
P. Merlatti \\ NORDITA \\ Blegdamsvej 17, 2100 Copenhagen $\varnothing$, Denmark \\ merlatti@nbi.dk
}

\begin{abstract}
I consider supergravity solutions of D5 branes wrapped on supersymmetric 2-cycles and use them to discuss relevant features of four-dimensional $\mathcal{N}=1$ super YangMills theories with gauge group $S U(N)$. In particular, using a gravitational dual of the gaugino condensate, it is shown that is possible to obtain the complete NSVZ $\beta$-function. It is also described how different aspects of the gauge theory are nicely encoded in this supergravity solution.
\end{abstract}

Proceedings of the RTN-Workshop "The quantum structure of spacetime and the geometric nature of fundamental interactions", Leuven, September 2002 


\section{The gauge gravity correspondence and wrapped branes}

Recently, it has become more and more evident that a lot of relevant information about supersymmetric Yang-Mills (SYM) theories can be obtained by studying their dual supergravity backgrounds produced by stacks of D branes. This gauge/gravity correspondence has been throughly investigated in different cases. In this contribution I will mainly concern with $\mathcal{N}=1$ gauge theory in four dimensions. One way to get this amount of supersymmetry is to consider D branes whose world-volume is partially wrapped on a supersymmetric cycle inside a Calabi-Yau space. The unwrapped part of the brane world-volume remains flat and supports a gauge theory. In order to preserve some supersymmetry, the normal bundle to the wrapped D branes has to be partially twisted and, as a consequence of this twist, some world-volume fields become massive and decouple. This procedure has been used by Maldacena and Nuñez in Ref. [1 to study the pure $\mathcal{N}=1$ SYM theory in four dimensions. This is obtained by considering the world-volume theory of the D5-branes at energies where both the higher string modes as well as the KK excitations on the 2-cycle decouple. The back-reaction of the D-branes deforms the original background. The topology of the resulting space is in general very different from the starting CY space. In this case, as discussed by Vafa in Ref. 3, one expects the resulting space be a deformed CY space, where the 2-cycle has shrunk but a 3-sphere has blown-up, rendering a ten-dimensional non-singular solution. As it is the case for gravity duals of confining gauge theories, one cannot obtain an exact duality since extra degrees of freedom, not belonging to the gauge theory, cannot be decoupled within the supergravity regime. The question is now whether one can extract information on the gauge theory from this dual supergravity background.

This question has been addressed in many papers [1, 2, 4, 5, 6, 7] and a number of information on the gauge theory have been shown to be predicted by the dual supergravity background in a precise and quantitative way. In particular, in this contribution I will show how to derive from the supergravity solution the perturbative running of the gauge coupling with the corresponding $\beta$-function, the chiral symmetry anomaly, the phenomenon of gaugino condensation with the corresponding breaking of the chiral symmetry to $\mathbb{Z}_{2}$ in the IR.

\subsection{Ten dimensional supergravity solution}

Let us start by summarizing the explicit form of the MN solution. This solution is obtained from a non-singular domain wall solution of seven-dimensional gauged supergravity [11, parameterized by coordinates $\left(x_{0}, \ldots, x_{3}, \rho, \theta_{1}, \phi_{1}\right)$, and uplifting to ten dimensions along a 3-sphere [8, 9], parameterized by coordinates $\left(\psi, \theta_{2}, \phi_{2}\right)$. The relevant fields (the metric, 
the dilaton and the RR 3-form the D5-branes magnetically couple to) are

$$
\begin{aligned}
d s^{2} & =e^{\Phi} d x_{1,3}^{2}+e^{\Phi} \alpha^{\prime} g_{s} N\left[e^{2 h}\left(d \theta_{1}^{2}+\sin ^{2} \theta_{1} d \phi_{1}^{2}\right)+d \rho^{2}+\sum_{a=1}^{3}\left(\sigma^{a}-A^{a}\right)^{2}\right] \\
e^{2 \Phi} & =\frac{\sinh 2 \rho}{2 e^{h}} \\
F^{(3)} & =2 \alpha^{\prime} g_{s} N \prod_{a=1}^{3}\left(\sigma^{a}-A^{a}\right)-\alpha^{\prime} g_{s} N \sum_{a=1}^{3} F^{a} \wedge \sigma^{a}
\end{aligned}
$$

where

$$
\begin{aligned}
A^{1} & =-\frac{1}{2} a(\rho) d \theta_{1}, \quad A^{2}=\frac{1}{2} a(\rho) \sin \theta_{1} d \phi_{1}, \quad A^{3}=-\frac{1}{2} \cos \theta_{1} d \phi_{1} \\
e^{2 h} & =\rho \operatorname{coth} 2 \rho-\frac{\rho^{2}}{\sinh ^{2} 2 \rho}-\frac{1}{4}, \quad a(\rho)=\frac{2 \rho}{\sinh 2 \rho}
\end{aligned}
$$

$A^{a}$ being the three $S U(2)_{L}$ gauge fields of the relevant seven-dimensional gauged supergravity. The $\sigma^{a}$ are the left-invariant one-form parameterizing the 3-sphere

$$
\begin{aligned}
\sigma^{1} & =\frac{1}{2}\left(\cos \psi d \theta_{2}+\sin \psi \sin \theta_{2} d \phi_{2}\right) \quad, \quad \sigma^{2}=-\frac{1}{2}\left(\sin \psi d \theta_{2}-\cos \psi \sin \theta_{2} d \phi_{2}\right) \\
\sigma^{3} & =\frac{1}{2}\left(d \psi+\cos \theta_{2} d \phi_{2}\right)
\end{aligned}
$$

\section{$2 \mathcal{N}=1$ super Yang-Mills theories in 4 dimensions}

We now show how to use the supergravity solution described in the previous paragraph to extract information on the corresponding gauge theory. To this aim, we consider the world-volume action of a wrapped five-brane with a gauge field strength $F$ and then extract from it the quadratic terms in $F$. The non abelian bosonic action that we obtain is then simply [4]:

$$
S_{Y M}=-\frac{1}{4 g_{\mathrm{YM}}^{2}} \int d^{4} x F_{\alpha \beta}^{A} F_{A}^{\alpha \beta}+\frac{\theta_{\mathrm{YM}}}{32 \pi^{2}} \int d^{4} x F_{\alpha \beta}^{A} \widetilde{F}_{A}^{\alpha \beta}
$$

where

$$
\begin{aligned}
\frac{1}{g_{\mathrm{YM}}^{2}} & =\frac{\tau_{5}(2 \pi)^{2} \alpha^{\prime 2}}{2} \int_{S^{2}} \mathrm{e}^{-\Phi} \sqrt{-\operatorname{det} G} \\
\theta_{\mathrm{YM}} & =\tau_{5}(2 \pi)^{4} \alpha^{\prime 2} \int_{S^{2}} C^{(2)}
\end{aligned}
$$

where $C^{(2)}$ is the RR two form potential.

In the above set of coordinates, $\left(\theta_{1}, \Phi_{1}, \theta_{2}, \Phi_{2}, \psi\right)$, the actual $S^{2}$ that enters these gauge-gravity relations is [2]

$$
S^{2}: \theta_{1}=-\theta_{2} \quad, \quad \phi_{1}=-\phi_{2} \quad, \quad \psi=0
$$


Note that the inverse square of the YM coupling constant is proportional to the volume of the 2-sphere on which the D5 branes are wrapped and the YM vacuum angle is proportional to the flux of the R-R 2-form across this 2-sphere.

If we insert the explicit form of the supergravity solution (11)-(5) in eq.(8) (9), after simple calculations we obtain

$$
\frac{1}{g_{\mathrm{YM}}^{2}}=\frac{N}{16 \pi^{2}} Y(\rho) \quad \theta_{\mathrm{YM}}=-N \psi_{0}
$$

where

$$
Y(\rho)=4 e^{2 h(\rho)}+(a(\rho)-1)^{2}=4 \rho \tanh \rho, \quad a(\rho)=\frac{2 \rho}{\sinh 2 \rho},
$$

$\psi_{0}$ being an integration constant.

In the following we will exploit these results to discuss some relevant features of the pure $\mathcal{N}=1 \mathrm{SYM}$ theory from the gravitational point of view.

\subsection{The chiral symmetry}

As it is well-known, the $\mathcal{N}=1$ SYM theory possesses a classical abelian $U(1)_{R}$ symmetry which becomes anomalous at the quantum level. If we assign $R$-charge 1 to the gaugino $\lambda(x)$ so that under a chiral transformation with parameter $\varepsilon$

$$
\lambda(x) \rightarrow \mathrm{e}^{\mathrm{i} \varepsilon} \lambda(x)
$$

then the presence of the anomaly implies that

$$
\theta_{\mathrm{YM}} \rightarrow \theta_{\mathrm{YM}}-2 N \varepsilon
$$

From eq.(11) it is easy to see that we are describing the gauge theory in a fixed $\theta$ vacuum. Following a suggestion made in [1] we now argue that the other solutions are related to this by a seven dimensional gauge transformation, namely

$$
A^{\prime}=\mathrm{e}^{\mathrm{i} \varepsilon \sigma_{3}} A \mathrm{e}^{-\mathrm{i} \varepsilon \sigma_{3}}
$$

As pointed out in [2], different seven dimensional gauge choices correspond to different parametrizations of the relevant ten dimensional geometry. In the case at hand, after the gauge transformation (15), the longitudinal $S^{2}$ which enters the various gauge-gravity relations is now

$$
S^{2}: \theta_{1}=-\theta_{2} \quad, \quad \phi_{1}=-\phi_{2} \quad, \quad \psi=2 \varepsilon
$$

From (8,9) it is easy to see that, on the gauge theory side, we are now describing the same running of the gauge coupling (as in (11) ) but we are studying the theory in a different $\theta$-vacuum, namely:

$$
\theta_{\mathrm{YM}}^{\prime}=-N\left(\psi_{0}+2 \varepsilon\right)
$$

The new form of the solution seems to suggest that even the phase of the gaugino condensate (that we identify with the supergravity field $a(\rho)$, see next section and [6, 4]) 
has been shifted by $2 \varepsilon$. We are then describing the different vacua related by the $U(1)_{R}$ symmetry of the gauge theory.

The $U(1)_{R}$ transformation which relates the different vacua is

$$
\psi \rightarrow \psi+2 \varepsilon
$$

This transformation is not a symmetry of the supergravity solution (11)-(3) (or of one related to this by the gauge transformation (15)) and is not even an isometry of the metric. However, there is a region where the transformation (18) is an isometry, namely the large$\rho$ region where the function $a(\rho)$ becomes exponentially small and can be neglected. In fact, if we remove $a$, then all explicit $\psi$ dependence disappears from the metric (11) and the parametrization of the world-volume 2-cycle no longer fixes the value of $\psi$ (differently from (10, 16) $\psi$ can be now an arbitrary constant). The $\psi$ dependence instead still remains in the R-R 2-form (3), which therefore is not invariant under (18). However the relevant quantity that should be considered is the flux of $C^{(2)}$ across the 2-sphere, i.e.

$$
\left.\frac{1}{4 \pi^{2} \alpha^{\prime} g_{s}} \int_{S_{2}} C^{(2)}\right|_{a=0}=-\frac{N}{2 \pi}\left(\psi-\psi_{0}\right)
$$

which is allowed to change by integer values. Thus the transformations (18) with $\varepsilon=$ $(\pi / N) k$ and $k$ integer are true symmetries of the supergravity background in the large- $\rho$ region. These are precisely the non-anomalous $\mathbf{Z}_{2 N}$ transformations of the gauge theory that correspond to shifts of the $\theta$-angle by integer multiples of $2 \pi$. What we have described here is therefore the gravitational counterpart of the well-known breaking of $U(1)_{R}$ down to $\mathbf{Z}_{2 N}$.

However, in the true supergravity solution $a(\rho)$ is not vanishing and thus even the nonanomalous $\mathbf{Z}_{2 N}$ transformations are not symmetries of the background. Thus, the only non-anomalous symmetries of the supergravity background are given by (18) with $\varepsilon=k \pi$ and $k$ integer. This phenomenon is the gravitational counterpart of the spontaneous breaking of the chiral symmetry from $\mathbf{Z}_{2 N} \rightarrow \mathbf{Z}_{2}$.

\subsection{The gaugino condensate}

In the previous subsection we have seen that the presence of a non-vanishing $a(\rho)$ in the supergravity solution is responsible for the spontaneous chiral symmetry breaking to $\mathbf{Z}_{2}$, which, on the gauge theory side, is accompanied by the presence of a non-vanishing

value of the gaugino condensate $\left\langle\lambda^{2}\right\rangle \equiv\left\langle 0\left|\left(\frac{\operatorname{Tr} \lambda^{2}}{16 \pi^{2}}\right)\right| 0\right\rangle$. Thus, it appears very natural to conjecture that the gravitational dual of this condensate is precisely the function $a(\rho)$ that is present in the supergravity solution (11)-(3) [6, 4]

The gaugino condensate $\left\langle\lambda^{2}\right\rangle$ belongs to a class of gauge invariant operators which do not acquire any anomalous dimensions. This happens because the gaugino condensate is the lowest component of the so-called anomaly multiplet whose scale dimensions are protected by virtue of the fact that its top component is the trace of the energy-momentum 
tensor which is a conserved current. Thus, since the engineering dimension of $\left\langle\lambda^{2}\right\rangle$ is 3 , we have

$$
\left\langle\lambda^{2}\right\rangle=c \Lambda^{3}
$$

where $\Lambda$ is the (exact) dynamical scale of the $\mathcal{N}=1$ SYM theory and $c$ a computable constant.

In view of our previous discussion, we now propose to identify the function $a(\rho)$ given in (5) with the gaugino condensate $\left\langle\lambda^{2}\right\rangle$ measured in units of the (arbitrary) mass scale $\mu$ that is introduced to regulate the theory. Thus, we write [4]

$$
a(\rho)=\frac{\Lambda^{3}}{\mu^{3}}
$$

This crucial equation allows us to establish a precise relation between the supergravity radial coordinate $\rho$ and the scales of the gauge theory. Notice that, as usual, since $a(\rho) \rightarrow 0$ for $\rho \rightarrow \infty$, the large- $\rho$ region corresponds to the UV regime of the gauge theory, and vice-versa the small- $\rho$ region corresponds to the IR regime.

Now we exploit the relation (21) to compute the perturbative $\beta$-function of the pure $\mathcal{N}=1$ SYM theory.

\subsection{The beta function}

From the above equations one can get the complete perturbative $\mathcal{N}=1 \beta$-function. We can write

$$
\beta\left(g_{\mathrm{YM}}\right)=\frac{\partial g_{\mathrm{YM}}}{\partial \ln (\mu / \Lambda)}=\frac{\partial g_{\mathrm{YM}}}{\partial \rho} \frac{\partial \rho}{\partial \ln (\mu / \Lambda)}
$$

and compute the two derivative contributions from eq. (11) and eq. (21), respectively. In doing so, let us first disregard the exponential corrections, which are sub-leading at large $\rho$ and which give rise to non-perturbative contributions. We easily get

$$
\frac{\partial g_{\mathrm{YM}}}{\partial \rho}=-\frac{N g_{\mathrm{YM}}^{3}}{8 \pi^{2}}, \quad \frac{\partial \rho}{\partial \ln (\mu / \Lambda)}=\frac{3}{2}\left(1-\frac{1}{2 \rho}\right)^{-1}=\frac{3}{2}\left(1-\frac{N g_{\mathrm{YM}}^{2}}{8 \pi^{2}}\right)^{-1}
$$

The final result is then

$$
\beta\left(g_{\mathrm{YM}}\right)=-3 \frac{N g_{\mathrm{YM}}^{3}}{16 \pi^{2}}\left(1-\frac{N g_{\mathrm{YM}}^{2}}{8 \pi^{2}}\right)^{-1}
$$

which is the NSVZ $\beta$-function [10].

Notice that the complete supergravity solution suggests the presence of a Landau pole. However, this is not really an issue. The curvature of the MN background goes like $\alpha^{\prime} \mathcal{R} \sim 1 / g_{s} N$ so the regime in which the supergravity approximation is reliable is for large $N$. In this regime a Landau pole can indeed be present even if the gauge coupling remains finite at the scale $\Lambda$, since in eq. (11) it is really $g_{\mathrm{YM}}^{2} N$ which is going to infinity for $\rho \rightarrow 0(\mu \rightarrow \Lambda)$ and not the gauge coupling itself. To discuss the duality in the deep IR at finite $N$, one has to go beyond the supergravity approximation. 


\section{Conclusions and Perspectives}

We have shown that many interesting features of the pure $\mathcal{N}=1 \mathrm{SYM}$ theories in four dimensions are quantitatively encoded in the supergravity solutions that describe D5 branes wrapped on supersymmetric 2-cycles. These features comprise the running of the gauge coupling constant, the $\beta$-function and the chiral anomaly.

All these are well known results on the gauge theory side, but the important point is that they are nicely encoded in a classical supergravity solution. Moreover, I find particularly nice that the gauginos condensation (a typical infrared phenomenum of the gauge theory) is also described by this supergravity solution.

Another issue related to the infrared of the gauge theory is that of fractional instantons. The supergravity solution seems to suggest that they modify the running of the YangMills coupling constants itself [4]. However in the IR these fractional instantons mix with some other unwanted Kaluza-Klein effects on the gravity side. This renders the correspondence with the gauge theory difficult to establish. Nevertheless one could infer that fractional instantons with topological charge $2 / N$ modify the running of the coupling constant, giving a modified $\beta$-function with respect to the NSVZ one. This would avoid the pole of the NSVZ $\beta$-function, whose physical interpretation is not complety clear, giving:

$$
\beta\left(g_{\mathrm{YM}}\right)=-3 \frac{N g_{\mathrm{YM}}^{3}}{16 \pi^{2}}\left(\operatorname{coth}\left(\frac{8 \pi^{2}}{N g_{\mathrm{YM}}^{2}}\right)-\frac{N g_{\mathrm{YM}}^{2}}{8 \pi^{2}}\right)^{-1}
$$

It is surely interesting to look for a precise form of the correspondence also in the infrared of the gauge theory.

Acknowledgements: I thank M. Bertolini, P. Di Vecchia, A. Lerda and W. Mueck for usefull discussions and/or correspondence.

I would like also to thank the organizers of the workshop "The quantum structure of spacetime and the geometric nature of fundamental interactions" in Leuven for the stimulating and nice atmosphere I have found there.

Work supported by the European Community's Human Potential Programme under contract HPRN-CT-2000-00131 Quantum Spacetime in which P.M. is associated to Copenhagen.

\section{References}

[1] J. Maldacena and C. Nuñez, Toward The Large $N$ Limit Of N=1 Super Yang Mills, Phys. Rev. Lett. 86 (2001) 588, hep-th/0008001.

[2] M. Bertolini and P. Merlatti, arXiv:hep-th/0211142

[3] C. Vafa, Superstrings and topological strings at large N, J. Math. Phys. 42 (2001), 2798, hep-th/0008142. 
[4] P. Di Vecchia, A. Lerda and P. Merlatti, $N=1$ and $N=2$ super Yang-Mills theories from wrapped branes, hep-th/0205204.

[5] A. Loewy and J. Sonnenschein, On The Holographic Duals Of $\mathcal{N}=1$ Gauge Theories, JHEP 0108 (2001) 007, hep-th/0103163

[6] R. Apreda, F. Bigazzi, A.L. Cotrone, M. Petrini, A. Zaffaroni, Some comments on N=1 gauge theories from wrapped branes, Phys. Lett. B536 (2002) 161, hep-th/0112236.

[7] P. Olesen and F. Sannino, $N=1$ super Yang-Mills from supergravity: The UV-IR connection, hep-th/0207039.

[8] M. Cvetic, H. Lu and C.N. Pope, Consistent Kaluza-Klein sphere reductions, Phys. Rev. D62 (2000) 064028, hep-th/0003286

[9] A.H. Chamseddine and W.A. Sabra, $D=7 S U(2)$ gauged supergravity from $D=10$ supergravity, Phys. Lett. B476 (2000) 415, hep-th/9911180

[10] V. Novikov, M. Shifman, A. Vainstein and V. Zakharov, Exact Gell-Mann-Low Function Of Supersymmetric Yang-Mills Theories From Instanton Calculus, Nucl. Phys. B229 (1983) 381.

[11] A.H. Chamseddine and M.S. Volkov, Non-abelian solitons in N=4 gauge supergravity, Phys. Rev. Lett. 79 (1997) 3343, hep-th/9707176 